\title{
Surgical Resection of Idiopathic Distal Anterior Choroidal Artery Aneurysm Assisted by Frameless Stereotactic System Guidance: Case Report and Literature Review
}

\author{
Feng YE¹, Chuanyuan $\mathrm{TAO}^{2}$, Shenghua XIONG³ , Chao YOU² \\ ${ }^{1}$ People's Hospital of Deyang City, Department of Neurosurgery, Deyang, Sichuan, China \\ ${ }^{2}$ West China Hospital, Sichuan University, Department of Neurosurgery, Chengdu, Sichuan, China \\ ${ }^{2}$ West China Hospital, Sichuan University, Department of Pathology, Chengdu, Sichuan, China
}

\section{ABSTRACT}

Idiopathic distal anterior choroidal artery (AChoA) aneurysms are particularly rare. Their diagnosis and treatment are difficult because of the particular characteristic of pathological anatomy. Our case mainly presented with intraventricular hemorrhage. His idiopathic aneurysm was located in the trigone of the undilated ventricle. Computed tomographic angiography demonstrated both the precise aneurysm and parent artery even when there was no dilated parent artery for the first time. It is also the first time this tiny aneurysm was resected assisted by frameless stereotactic guidance during operation after absorption of intraventricular blood. Computed tomographic angiography and frameless stereotactic guidance benefit the diagnosis and treatment of this kind of aneurysm. Sacrificing plexual AChoA did not cause any neurologic deficit.

KEYWORDS: Aneurysm, Anterior choroidal artery, Distal, Intraventricular hemorrhage, Surgery

\section{INTRODUCTION}

I diopathic distal anterior choroidal artery (AChoA) aneurysms are quite rare; only 10 cases confirmed by cerebral angiography have been reported in the English literature $(10-12,21,22,24,26,32,33)$. Their diagnosis and treatment strategy are totally different from the proximal AChoA aneurysms because of the particular characteristics of pathological anatomy. Herein, we report a rare case located in the trigone of the ventricle. It was the first time this tiny aneurysm was resected assisted by frameless stereotactic guidance during operation after absorption of intraventricular blood. A thorough review of pertinent English literature has also been performed. The study was approved by the biological and medical ethics committee of our hospital. Written informed consent was obtained from the patient.

\section{CASE REPORT}

A 23-year-old man experienced a sudden onset of severe headache, nausea, and vomiting on June 15, 2013. His medical history was insignificant and without head trauma, infection or hypertension. This patient was sent to a local hospital urgently. Immediate computed tomography (CT) of the brain disclosed a diffuse intraventricular hemorrhage (IVH), mainly in the right lateral ventricle, associated with a $10 \mathrm{~mm}$ intracerebral hemorrhage $(\mathrm{ICH})$ near the right posterior limb of internal capsule (Figure 1A). There was no subarachnoid hemorrhage or hydrocephalus. The patient underwent conservative management and his symptoms relieved gradually. 14 days later, he was transferred to our center for further investigation. On admission, he was alert and absent of any neurological deficit. Routine hematological, biochemical examinations, electrocardiogram and chest $\mathrm{x}$-ray were normal. Repeated CT revealed the absorption of IVH (Figure 1B). However, a nodular lesion was detected on magnetic resonance imaging (MRI) 
scans in the right trigone (Figures 1C, D). Further computed tomographic angiography (CTA) was performed suggesting the nodule was a small aneurysm measuring $1.8 \times 2.2 \mathrm{~mm}$ arising from the distal portion of the right AChoA (Figures 2A, $B)$. Digital subtraction angiography (DSA) clearly confirmed this saccular aneurysm located at the plexual segment distal to the choroidal fissure (Figures 2C, D). No other abnormality was documented. This aneurysm was regarded to be the source of the hemorrhage.

Twenty-four days after the onset, we performed a small right occipito-temporal craniotomy assisted by frameless stereotactic guidance. The right trigone was entered under the microscope. During operation, the field of the ventricle was clear without the inference of intraventricular blood. The choroid plexus was found to be normal. After partial resection of the choroid plexus, a saccular aneurysm $4 \times 4 \times 5 \mathrm{~mm}$ in size was exposed and then resected successfully without preserving the parent artery. Histological examination revealed the aneurysm to be a true one (Figure 3).

The postoperative course was uneventful. Head MRI and CTA performed 6 days after surgery indicated the complete excision of the aneurysm without hemorrhagic or ischemic complications (Figures 4A, B). The patient was discharged without any neurological deficit. Three-month follow-up DSA demonstrated the complete obliteration of the aneurysm (Figures 4C, D). The patient remained neurologically normal at 1-year follow-up.

\section{DISCUSSION}

Proximal AChoA aneurysms, accounting for $2-5 \%$ of intracranial aneurysms, usually occur at the junction of the internal carotid artery (ICA) and the AChoA (7). Distal AChoA aneurysms are rare (12). Only 35 cases confirmed by cerebral angiography were reported in the English literature from 1950 to July 2014 (1-6,9-26,28-33). They were mostly associated with Moyamoya disease (14/35) $(4,9,13-15,17-19,29,31)$, while others were associated with atherosclerosis $(4 / 35)(2,16,23,25)$, dissection $(2 / 35)(20,28)$, arteriovenous malformation $(2 / 35)$ $(3,31)$, iatrogenicity $(1 / 35)(6)$, infection $(1 / 35)(1)$, or a history of trauma (1/35) (5). However, nearly one third (10/35) were of idiopathic origin $(10-12,21,22,24,26,32,33)$. There is still some controversy for the time and strategy for the surgery of distal AChoA aneurysms. Unlike the distal AChoA aneurysms associated with Moyamoya disease reported recently to be treated endovascularly $(4,15,31)$, surgery is still the only choice for these idiopathic distal AChoA aneurysms. Our case

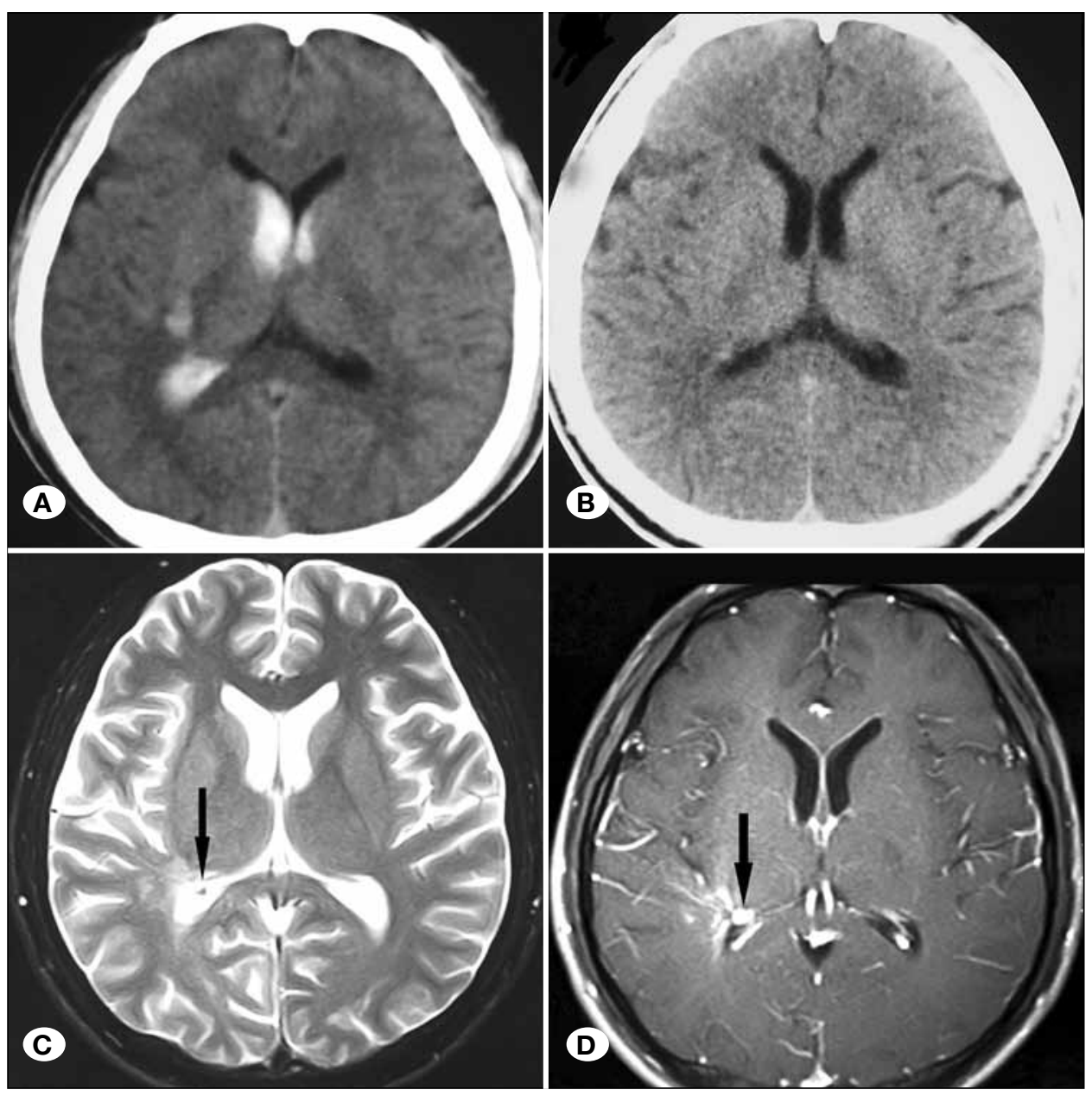

Figure 1: Preoperative imaging: A) CT showed IVH and a small ICH. B) Repeated CT before surgery revealed the absorption of $\mathrm{IVH}$. C, D) MRI showed signal abnormality (arrow) in the right trigone. 

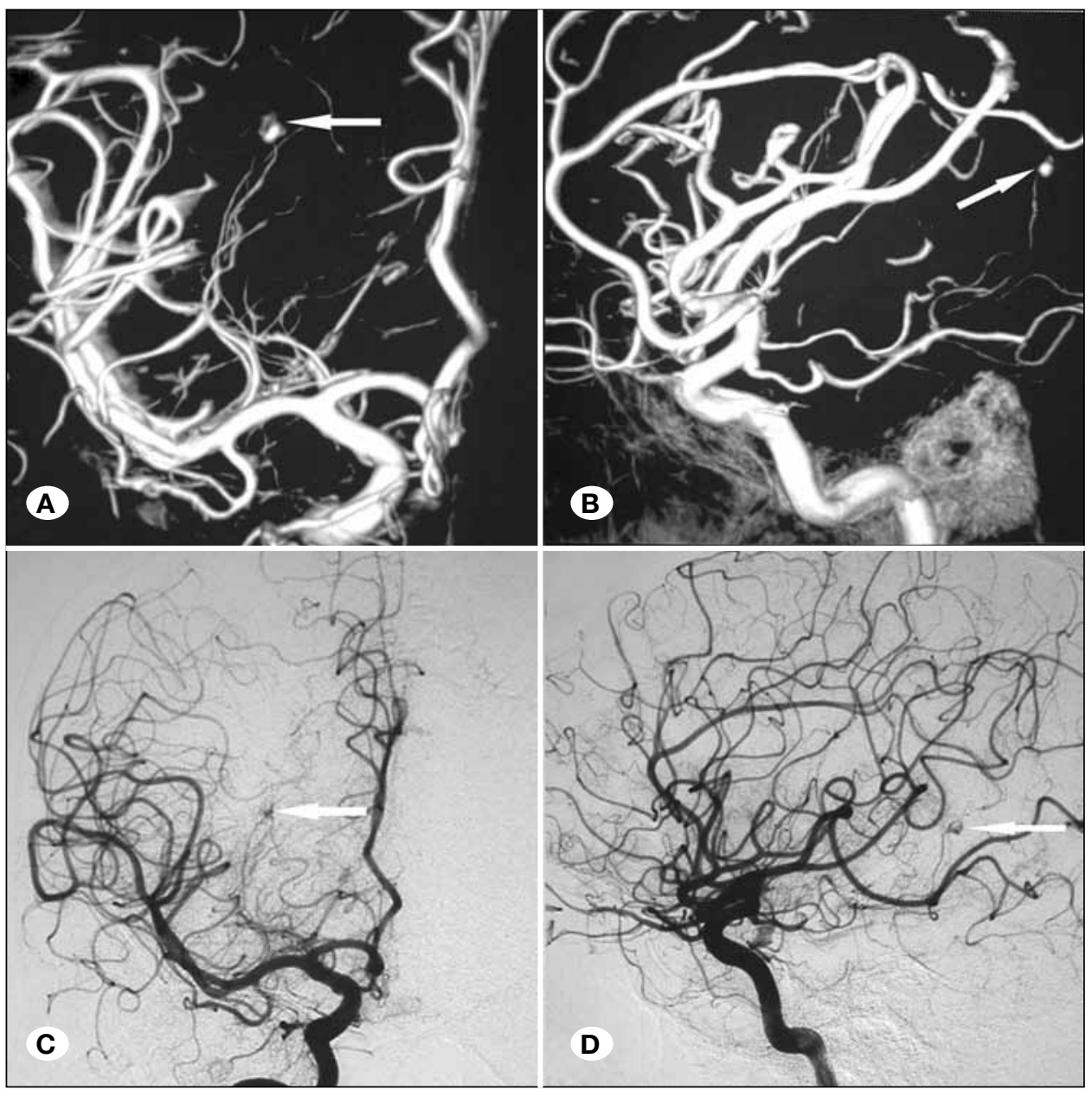

Figure 2: Preoperative imaging: A) (Frontal view), B) (Lateral view) CTA suggested a small aneurysm (arrow) on the distal portion of right AChoA. C) (Frontal view),

D) (Lateral view) DSA confirmed this plexual saccular aneurysm (arrow) distal to the choroidal fissure.

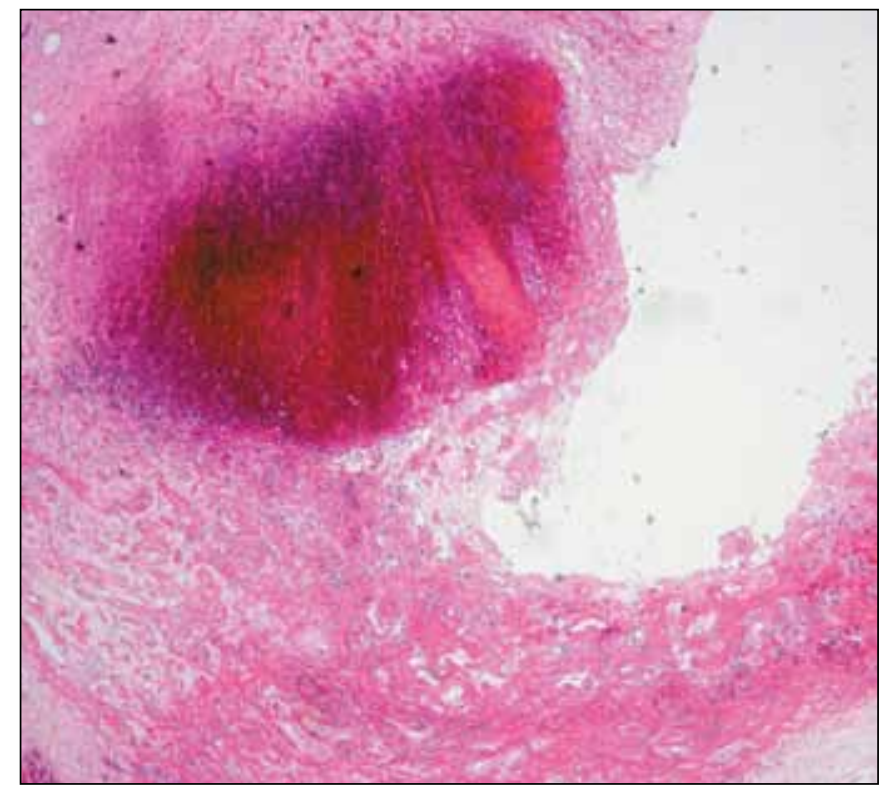

Figure 3: Histopathological photomicrograph revealed the mediomuscle layer and the internal elastic lamina of the aneurysmal wall was absent. Thrombosis and its organization were inside the aneurysmal cavity. There was no evidence of tumor or infection $(\mathrm{HE}$, original magnification, $\times 40)$. proved to be idiopathic. The clinical data of all these idiopathic cases including our case are summarized in Table I.

The incidence of primary IVH associated with $\mathrm{ICH}$ among all the patients with intracranial hemorrhage is $9 \%$ and most of them had associated hydrocephalus (58\%) (8). Unfortunately, most of the ruptured idiopathic distal AChoA aneurysms presented with IVH $(7 / 10)(11,12,21,24,26,32,33)$. It often lead to hydrocephalus or ventricular extension $(5 / 7)$ $(11,24,26,32,33)$, and emergency ventricular drainage was often needed to decrease the increased intracranial pressure consequently (32). It is difficult to differentiate IVH caused by ruptured idiopathic distal AChoA aneurysm from primary IVH because of the absence of any clues, such as Moyamoya disease, atherosclerosis, arteriovenous malformation or a history of trauma. Moreover, in a few reported cases, distal AChoA aneurysm could not be detected on initial cerebral angiography due to its small size (26). Therefore, the immediate increased transmural pressure gradient effect induced by insertion of a ventricular catheter appeared dangerous due to the high risk of re-bleeding caused by such missed intraventricular aneurysms. CTA has been performed in two idiopathic cases and only one aneurysm was detected but its precise parent artery was not determined (12). 


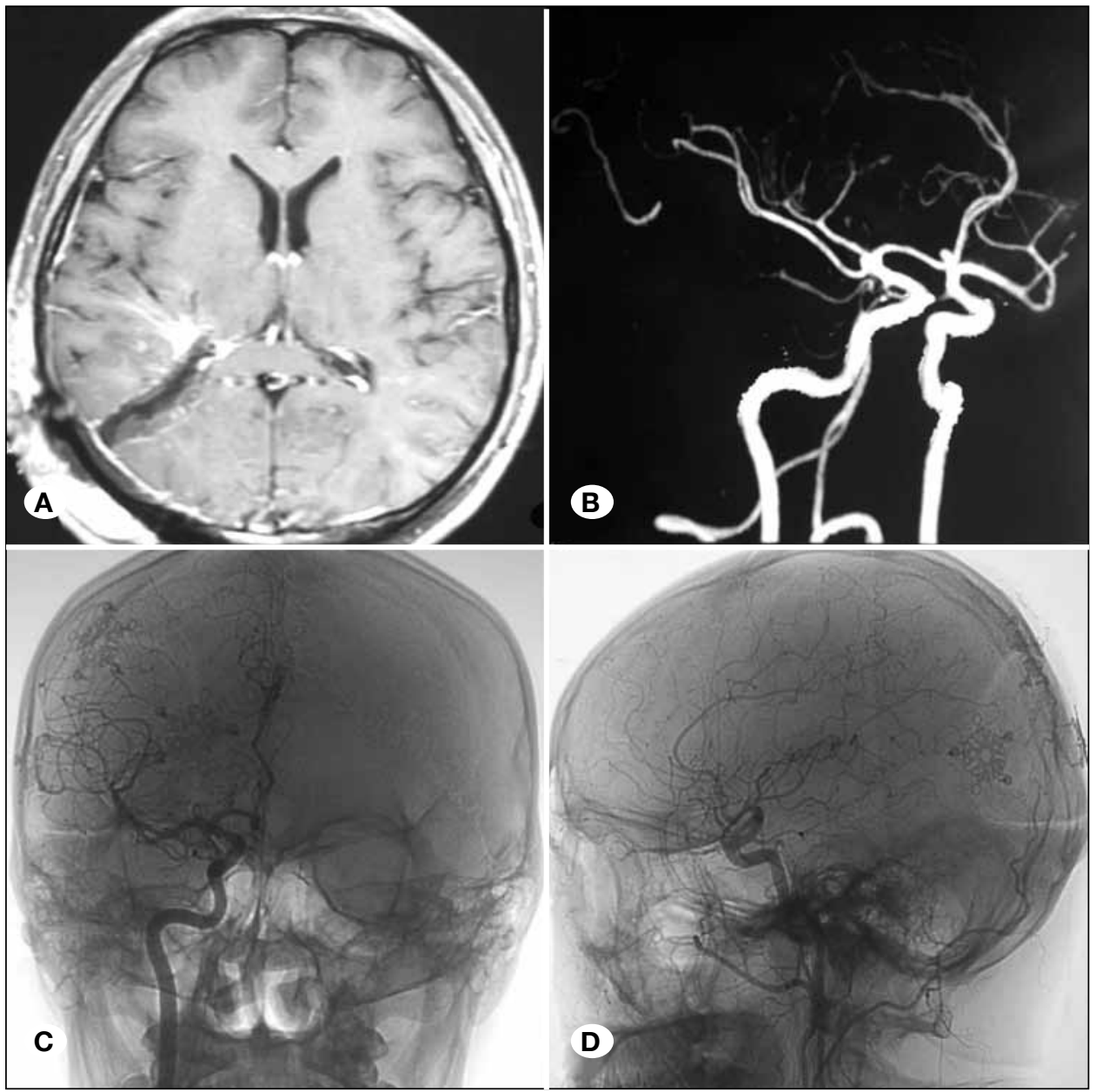

Figure 4: Postoperative imaging: A) MRI indicated no hemorrhagic or ischemic complications.

B) Postoperative CTA showed disappearance of the aneurysm. Three-month follow-up DSA confirmed complete obliteration of the aneurysm. C) Frontal view, D) Lateral view.
In our case, CTA demonstrated both the precise aneurysm and parent artery even when there was no dilated parent artery. The gold standard is still conventional angiography for diagnosis of these small aneurysms; however, we think CTA should be a valuable supplement examination for this kind of unexplained IVH, especially as a non-invasive choice when the initial cerebral angiography is negative or before emergency ventricular drainage.

The AChoA is divided into cisternal and plexal segments by the choroidal fissure (27). The cisternal segment, which extends from the origin of AChoA to the choroidal fissure, has a few vital branches (27). The plexual segment begins at the choroidal fissure and usually continues posteriorly within the temporal horn as a single trunk and mainly supplies the lateral ventricular choroid plexus, rarely as far forward as the foramen of Monro (27). No aneurysm associated with Moyamoya disease arose at the cisternal segment (4,9,13-15,17$19,29,31)$, but there are as many as three idiopathic aneurysms that developed at this segment in this review $(10,21,22)$. In these cases, only one patient, who presented with slight ischemic symptoms, received conservative treatment (22). The other two aneurysms were both clipped via the opened sylvian fissure with the cisternal segment preserved similar to the treatment of proximal AChoA aneurysms, because one or more cisternal branches' occlusion may result in severe neurological consequences $(10,21)$. Plexual aneurysms present some unique problems. With the progress of DSA and endovascular technique, four plexual AChoA aneurysms associated with Moyamoya disease have been treated successfully by endovascular embolization recently $(4,15,31)$. However, maybe it partly benefited from the compensatory dilation of AChoA (4,31). All the seven cases of idiopathic plexual $A C h o A$ aneurysm were located in the temporal horn of the lateral ventricle $(11,12,24,26,32,33)$. So far, no endovascular embolization has been performed for these idiopathic distal AChoA aneurysms because of the worry of occlusion in the cisternal segment. Only one of them died from a thrombosis of the pulmonary artery before the operation (11). All the other six plexual aneurysms were clipped or/and resected via the transtemporal-ventricular $(5 / 7)(24,12,26,33)$ or transtemporooccipito-trigone approach (1/7) (32) with or without proximal control, because the minimal hemorrhage could be easily controlled with bipolar cautery even when the aneurysm ruptured accidentally during operation (12) and sacrificing the plexual segment does not usually cause any neurologic deficit (27).

In this report, the small plexual AChoA aneurysm was located in the trigone of the undilated ventricle without obvious 


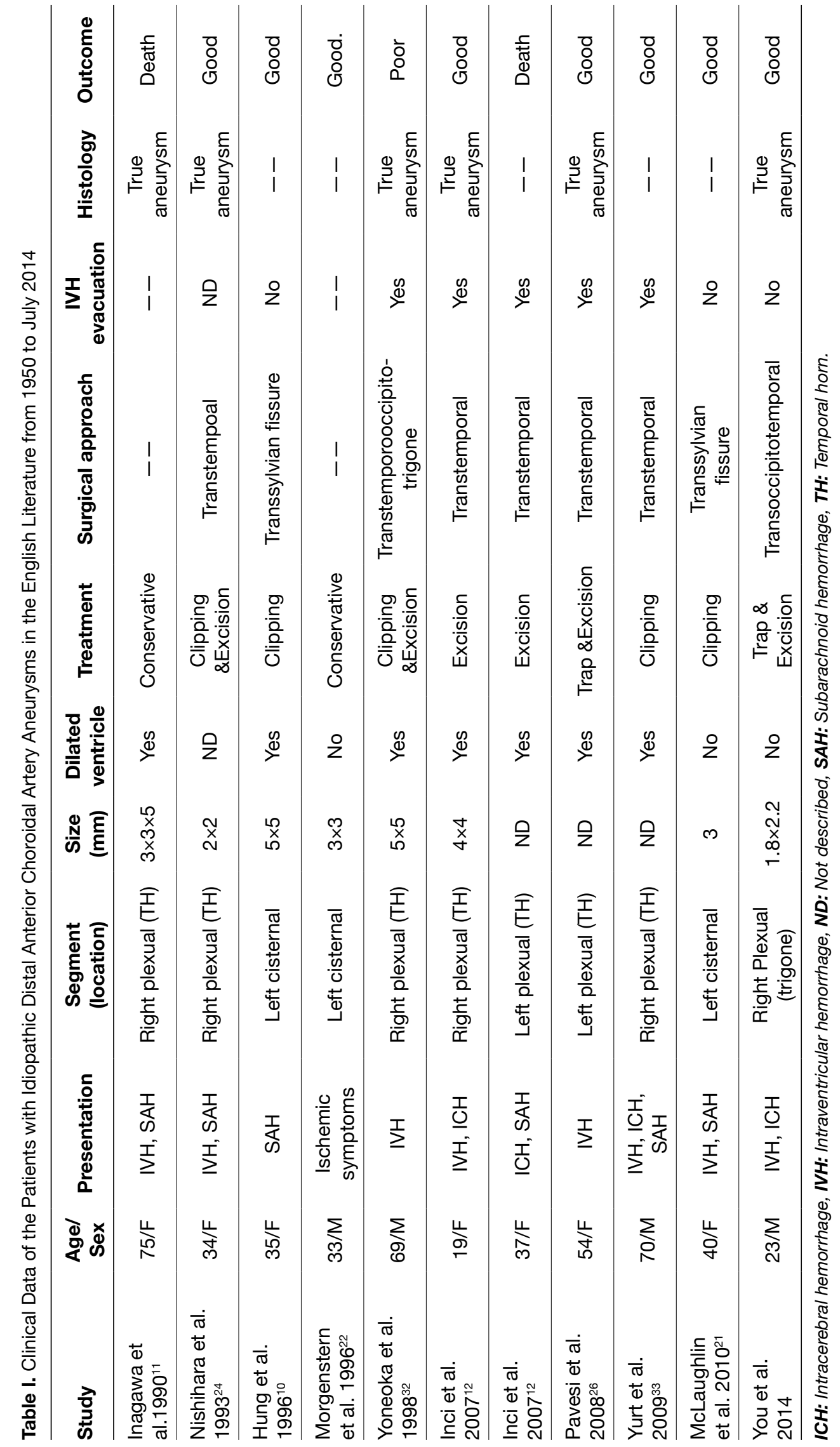


dilated AChoA. This is the first idiopathic distal AChoA aneurysm reported located in the trigone, and we performed a small right occipito-temporal craniotomy assisted by frameless stereotactic guidance for the first time. The different approaches described above have complications in proportion to the extent of the resection of brain tissue. The choice of surgical approach for our patient was a challenge. Detection of such small aneurysms during operation may be difficult within the undilated trigone. Although the clotting may be helpful for the location of the top of the aneurysm, the evacuation of hematoma was performed in at least $5(5 / 7)$ cases of the operated idiopathic plexual AChoA aneurysms during the operation $(12,26,32,33)$, and the suction system may aspirate the small aneurysm (32). Our operation was performed after the absorption of intraventricular blood. With the aid of frameless stereotactic guidance, the entry and target points were chosen by determining a linear trajectory through the scalp leading to the aneurysm with the least amount of dissection and brain retraction. Without the intraventricular blood, the aneurysm, surrounding choroid and vessels could be identified clearly. The aneurysm was resected without neurological deficit as it represented a surgical challenge to clip due to the small diameter of the parent vessel.

\section{CONCLUSION}

(1) CTA could be a valuable examination for the unexplained IVH, especially when the initial cerebral angiography is negative or before the emergency ventricular drainage. (2) So far, surgery is still the only choice for the idiopathic distal AChoA aneurysms. Using frameless stereotactic guidance helps to minimize the amount of dissection and brain retraction and locate the deep aneurysm without a dilated lateral ventricle. (3) A clear operation field after the absorption of intraventricular blood benefits the identification of a deep small aneurysm and its surrounding vessels. (4) Sacrificing plexual AChoA does not usually cause any neurologic deficit.

\section{ACKNOWLEDGEMENT}

We thank the patient who trusted us and all the staff who helped this study.

\section{- REFERENCES}

1. Ahn JY, Han IB, Hong CK, Joo JY: Ruptured distal anterior choroidal artery aneurysm. J Clin Neurosci 13: 872-875, 2006

2. Butler $A B$, Partain RA, Netsky MG: Primary intraventricular hemorrhage. Neurology 22: 675-686, 1972

3. Caram PC, Sharkley PC, Alvord Jr EC: Thalamic angioma and aneurysm of the anterior choroidal artery with intraventricular hematoma. J Neurosurg 17: 347-352, 1960

4. Choulakian A, Drazin D, Alexander MJ: NBCA embolization of a ruptured intraventricular distal anterior choroidal artery aneurysm in a patient with moyamoya disease. J Neurolntervent Surg 2: 368-370, 2010

5. Cressman MR, Hayes GJ: Traumatic aneurysm of the anterior choroidal artery. Case report. J Neurosurg 24: 102-141, 1966
6. Dolati P, Sutherland G, Wong J, Hudon M, Goyal M: Distal anterior choroidal artery aneurysm following iatrogenic posterior cerebral artery occlusion: A case report and review of literature. Acta Neurochir 154:53-57, 2012

7. Friedman J, Pichelmann M, Piepgras D, Atkinson J, Maher C, Meyer F, Hansen K: Ischemic complications of surgery for anterior choroidal artery aneurysms. J Neurosurg 94:565-572, 2001

8. Giray S, Sen O, Sarica FB, Tufan K, Karatas M, Goksel BK, Yerdelen D, Cekinmez M, Can U: Spontaneous primary intraventricular hemorrhage in adults: Clinical data, etiology and outcome. Turk Neurosurg 19(4):338-344, 2009

9. Hamada J, Hashimoto N, Tsukahara T: Moyamoya disesae with repeated intraventricular hemorrhage due to aneurysm rupture. Report of two cases. J Neurosurg 80: 328-331, 1994

10. Hung KS, Lee TC, Lui CC: Aneurysm of superior branch of anterior choroidal artery mimicking carotid bifurcation aneurysms. Case report. Acta Neurochir 138(12):1464-1467, 1996

11. Inagawa T, Matsuda $Y$, Kamiya $K$, Aoyama $H$, Nagasako $\mathrm{R}$, Yamamoto $\mathrm{M}$ : Saccular aneurysm of the distal anterior choroidal artery. Case report. Neurol Med Chir 30:498-502, 1990

12. Inci S, Arat A, Ozgen T: Distal anterior choroidal artery aneurysms. Surg Neurol 67:46-52, 2007

13. Kasamo S, Asakura T, Yamamoto Y, Kobayashi E: Unilateral moyamoya disease associated with multiple aneurysms. A case report and review of the literature. Neurol Med Chir (Tokyo) 24:30-34, 1984

14. Kawai K, Narita K, Nakayama H, Tamura A: Ventricular hemorrhage at an early stage of moyamoya disease. Case report. Neurol Med Chir (Tokyo) 37:184-187, 1997

15. Kim SH, Kwon O-Ki Jung CK, Kang HS, Oh CW, Han MH, Kim YS, Baik SK: Endovascular treatment of ruptured aneurysms of pseudoaneurysms on the collateral vessels in patients with moyamoya disease. Neurosurgery 65:1000-1004, 2009

16. Knuckey NW, Epstein MH, Haas R, Sparadeo F: Distal choroidal artery aneurysm. Intraoperative localization and treatment. Neurosurgery 22:1084-1087, 1988

17. Konishi Y, Kadowaki C, Hara M, Takeuchi K: Aneurysms associated with moyamoya disease. Neurosurgery 16:484491,1985

18. Lee JK, Lee JH, Kim SH, Lee MC: Distal anterior choroidal artery aneurysm in a patient with moyamoya disease: Case report. Neurosurgery 48:222-225, 2001

19. Leveque $M$, McLaughlin N, Laroche M, Bojanowski MW: Endoscopic treatment of distal choroidal artery aneurysm. J Neurosurgery 114:116-119, 2011

20. Matsuura H, Otawa Y, Suzuki M, Ogawa A: Dissecting aneurysm of the anterior choroidal artery: Angiographical and MR imaging findings. Surg Neurol 53:334-336, 2000

21. McLaughlin N, Bojanowski M: Ruptured aneurysm arising from the anterior choroidal artery's cisternal segment. Can J Neurol Sci 37:283-285, 2010

22. Morgenstern LB, Hankins LL, Grotta JC: Anterior choroidal artery aneurysm and stroke. Neurology 47:1090-1092, 1966 
23. Nishida A, Tokunaga K, Hishikawa T, Sugiu K: Date I: Endovascular coil embolization of a ruptured distal anterior choroidal artery aneurysm associated with ipsilateral middle cerebral artery occlusion. Neurol Med Chir (Tokyo) 51:716719,2011

24. Nishihara J, Kumon Y, Matsuo Y, Sakai S: A case of distal anterior choroidal artery aneurysm: Case report and review of the literature. Neurosurgery 32:834-837, 1993

25. Papo I, Salvolini U, Caruselli G: Aneurysm of the anterior choroidal artery with intraventricular hematoma and hydrocephalus. Case report. J Neurosurg 39: 255-260, 1973

26. Pavesi G, Amista P, Munari M, Gardiman MP: Intraventricular hemorrhage caused by peripheral anterior choroidal artery aneurysm rupture. A case report. Neuroradiol J 21:717-720, 2008

27. Rhoton AL Jr: The supratentorial arteries. Neurosurgery 51 Suppl 4:53-120, 2002

28. Shih P, Pinnaduwage T, Hu L, Spetzler RF: A pediatric patient with a dissecting thrombotic anterior choroidal artery aneurysm: Case report. Neurosurgery 67:2-E518, 2010
29. Wong GKC, Boet R, Poon W: Ruptured distal anterior choroidal artery aneurysm presenting with right intracerebral hematoma: Clipping aided by subpial uncal resection. J Clin Neurosci 10:689 - 691, 2003

30. Yanaka K, Tsuboi K, Fujita K, Aoki K, Takeuchi S, Anno I, Nose T: Distal choroidal artery aneurysm associated with an arteriovenous malformation. Intraoperative localization and treatment. Surg Neurol 53:546-551, 2000

31. Yang S, Yu JL, Wang HL, Wang B, Luo Q: Endovascular embolization of distal anterior choroidal artery aneurysm associated with moyamoya diseases. Interv Neuroradiol 16:433-441, 2010

32. Yoneoka Y, Ezuka I, Takai N, Oda T, Tamura T, Yamashita S: Ruptured distal anterior choroidal artery aneurysm presenting with casting intraventricular hemorrhage. Acta Neurochir 140:185-189, 1998

33. Yurt A, Turan Y, Ucar K, Camlar M, Oran I: Ruptured distal anterior choroidal artery aneurysm. J Clin Neurosci 16:132134, 2009 